\title{
La inteligencia artificial y su aplicación en la enseñanza
}

\section{Begoña Gross}

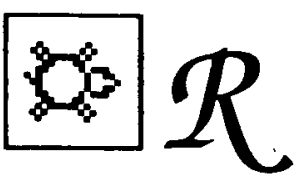

\section{Podríamos decir que un educador es una «inteligencia natural» que moviliza sus recursos cognitivos para enseñar lo mejor posible. ¿Hasta dónde ha llegado bay la ciencia en ese ambicioso objetivo de copiar la mente bumana? ¿Puede simularse la mente natural de un profesor experto y competente y situarla en un programa que eduque a través del ordenador? Este articulo introduce el tema y bace un balance de las posibilidades.}

El objetivo fundamental de este artículo es mostrar cómo las técnicas desarrolladas en inteligencia artificial (IA) son de gran utilidad para mejorar el software destinado al ámbito educativo. Para ello, en primer lugar, se realiza un breve resumen de las metas y objetivos generales de las investigaciones en I.A. realizadas hasta la fecha. Posteriormente se describen las diferentes aplicaciones de la I.A. en la educación dirigidas a los alumnos en tareas formativas e instructivas y a los profesores en tareas de diseño y planificación de las actividades docenctes. $\mathrm{El}$ artículo finaliza con una reflexión sobre las tendencias futuras de la inteligencia artificial aplicada a la educación.

\section{INTELIGENCIA ARTIFICIAL}

La expresión inteligencia artificial (IA) fue sugerida por J. McCarthy, profesor de la Universidad de Stanford, en 1956 para referirse a una parte de la informática dedicada al diseño de máquinas que fueran capaces de simular algunas de las conductas realizadas por el ser humano y que habitualmente catalogamos como inteligentes. Durante muchos años la investigación en IA fue llevada a cabo fundamentalmente en centros de investigación universitaria. Sólo a partir de los últimos diez años algunos de los resultados de estos estudios indicaron cómo muchos conceptos, procedimien- tos y técnicas desarrolladas en los laboratorios podían tener una valiosa aplicación en distintos terrenos tales como la industria, la medicina, las finanzas, etc. Cinco son las áreas más activas de investigación en IA: el lenguaje natural, la robótica, los interfaces de comunicación, la programación automática y los sistemas expertos. Explicaremos brevemente esta última pues es la que tiene en la actualidad una clara conexión con el desarrollo del software educativo.

El termino sistema experto no describe un producto sino más bien un conjunto de conceptos, procedimientos y técnicas que permiten utilizar la informática en una nueva dimensión. En esencia, los sistemas expertos permiten asistir en el análisis y resolución de problemas complejos. Intentan simular la conducta de un experto humano en un dominio específico de conocimiento. Un sistema experto debe pues contener todo el contenido que manejaría el experto y además, la forma de razonamiento utilizada por éste. Es decir, la manera en que el experto utiliza el conocimiento. Por ejemplo, si quisiéramos realizar un programa que enseñara a realizar operaciones aritméticas deberíamos introducir en el ordenador todos los conocimientos que posee un profesor especialista en esta temática (secuenciación de las operaciones, ideas sobre la motivación de los alumnos, organización del material, etc.) y además especificar los razo- 
namientos llevados a cabo por el profesor durante el proceso de enseñanza (cómo decide qué contenido transmitir, cómo corrige un determinado error, etc.). Así pues, con este tipo de programas se extiende el tipo de utilización de los ordenadores actuales ya que éstos no se centran únicamente en la realización de labores primordialmente repetitivas sino que se pasa a una utilización del ordenador como herramienta de ayuda en tareas complejas tales como el diagnóstico o la toma de decisiones. En este sentido, en el terreno de la industria y el comercio existen muchas esperanzas depositadas en este tipo de software. Nos obstante, es un tipo de producto que está comenzando a producirse. De hecho, su comercialización no comenzó hasta 1980 y, dada la complejidad del diseño de los mismos, la producción desde entonces ha sido bastante lenta y escasa. No obstante, existen varios ámbitos donde la producción ha sido más importante. No estamos refiriendo, fundamentalmente, a las aplicaciones financieras, la automatización de oficinas, los equipos de mantenimiento industrial y en el campo de la medicina. La aplicación en el terreno educativo, tal y como veremos más adelante, está aún en una fase experimental y todavía son muy escasos los productos existentes.

\section{TIPOS DE APLICACIONES DE LA I.A. EN LA EDUCACION}

Las aplicaciones educativas a partir del uso de técnicas de I.A. varían, básicamente, en función de los objetivos que se persiguen con el diseño del programa así como del tipo de conocimiento que la aplicación debe manipular.

Tal y como se muestra en la figura 1 podemos distinguir dos tipos generales: las que van dirigidas a proporcionar y facilitar un determinado aprendizaje al alumno y las que van dirigidas a ayudar al profesor en tareas tales como la planificación, el diseño y la organización de la tarea docente.

De entre las aplicaciones que permiten crear programas destinados a la enseñanza se pueden distinguir dos grandes áreas: los micromundos y los tutoriales inteligentes (TI). Ambas modalidades se encuentran enraizadas en dos posiciones diferentes de la utilización de la informática en el terreno educativo. La construcción de micromundos se sitúa en la búsqueda de herramientas que ayuden al sujeto a construir el conocimiento a partir de la interacción con el ordenador mediante procesos de aprendizaje basados fundamentalmente en el descubrimiento. En cambio, los TI al igual que el software propio de la enseñanza asistida por ordenador (EAO) tienen como objetivo la creación de programas que permitan transmitir un conocimiento previamente formalizado adoptando un tipo de conducta más «inteligente» que mejore el proceso de enseñanza a través de la máquina. $\mathrm{Pa}$ - samos a continuación a explicar más detenidamente cada una de ellas.

\section{MICROMUNDOS}

El concepto de micromundo fue utilizado por M. Minsky y S. Papert en 1971 al hacer referencia a una posible multitud de pequeñas fragmentaciones en las estrategias de resolución de problemas en dominios interactivos de aprendizaje. Posteriormente, Papert vuelve a utilizar dicho concepto en sus primeros trabajos sobre la construcción del lenguaje Logo (Papert, 1973).

El lenguaje Logo es un tipo de aplicación de la informática educativa que se diferencia del resto por no ser un programa previamente elaborado sino un lenguaje de programación que pretende, además, apartarse de los modelos clásicos de la E.A.O. No fue diseñado para que los niños aprendieran a programar. La finalidad de su uso va más allá de la adquisición de una cultura informática. Logo pretende ser una herramienta que facilite el desarrollo del sujeto y la construcción de aprendizajes mediante procesos de descubrimiento. No se trata pues de que el alumno aprenda mediante la recepción de informaciones emitidas por el ordenador con un programa previamente elaborado, sino de que el alumno programe el ordenador y esta actividad le permita reflexionar sobre sus estrategias de actuación construyendo así, de forma dinámica, sus propios aprendizajes.

Seymour Papert es la figura más representativa dentro de esta temática y es el principal impulsor del grupo que desarrolló este lenguaje. En 1969 creó el «Proyecto Logo» que tenía por objeto el desarrollo de un lenguaje de programación que pudiera ser utilizado en el ámbito escolar por niños de cualquier edad. En ese mismo año, apareció una primera versión del lenguaje Logo que se presentó como un dialecto de LISP y al igual que éste pretendía ser un lenguaje orientado hacia el procesamiento de listas que permitiera a los niños desarrollar un conocimiento más profundo de las ideas matemáticas y de las formas de aprendizaje. Sin embargo, Papert observó que la utilización de las listas implicaba un tipo de programación excesivamente compleja como para ser utilizada por los niños de cortas edades. Tomando como referencia las investigaciones y desarrollo de artefactos mecánicos de Grey Walter, diseñó la conocida tortuga mecánica cuyos movimientos básicos son transferidos posteriormente al ordenador mediante la creación de un entorno gráfico. Es a partir de la construcción de este entorno cuando Papert en. fatizó la utilización del concepto de micromundo situando a éste como objeto mediacional para el desarrollo del pensamiento formal. Así pues, podemos definir a un micromundo como «un subconjunto de la realidad, o de la realidad construida, cuya estructura es acorde con un mecanismo cognitivo determinado y puede suministrar un entor- 
no donde éste último pueda operar de forma efectiva» (L. Rodríguez-Roselló, 1986, p. 11).

A partir de estas primeras experiencias se fueron generando nuevos diseños del lenguaje más amplios y completos. En la actualidad está compuesto por cinco entornos diferentes de programación: gráfico (o geometría de la tortuga), procesamiento de listas, numérico, animación y música. Estos pueden trabajarse de forma independiente o, por el contrario, pueden realizarse programas utilizando el vocabulario específico de más de un entorno o de todos ellos.

De hecho, en el contexto de la utilización del lenguaje Logo, el concepto de micromundo posee en la actualidad dos sentidos diferentes; el procedente de la obra de Papert y el desarrollado por R. Lawler.

Papert $(1980,1988)$ concede a este concepto una visión bastante amplia. Para este autor un micromundo es un entorno de programación con la suficiente consistencia en sí mismo como para permitir la exploración de conocimientos muy diversos. Desde esta óptica la geometría de la tortuga es un claro ejemplo de micromundo el cual puede servir para explorar aspectos matemáticos, geométricos, físicos, espaciales, etc.

R. Lawler (1984) restringe mucho más el significado de este concepto y considera a un micromundo como un procedimiento o conjunto de procedimientos alrededor de alguna idea valiosa para desarrollar en el alumno el aprendizaje de un conocimiento o estrategia específica. Desde este punto de vista, un micromundo puede ser algo tan simple como un procedimiento para construir espirales, o algo mucho más complejo como el diseño de un programa con el que el sujeto pueda realizar acciones sobre diferentes tortugas probando reacciones físicas tales como la velocidad, la aceleración, etc.

Sea considerado en un sentido más amplio o restringido, la idea central viene a ser la misma. Es decir, la construcción de entornos abiertos que permitan al sujeto la exploración de ideas a través de la interacción con el ordenador. No hay una enmarcación previa de los conocimientos a transmitir, el alumno debe descubrirlos mediante su acción. El concepto de micromundo posee pues un modelo de aprendizaje fundamentado en la teoría piagetiana.

\section{La EAO y los TI: aspectos comparativos}

Una de las primeras y más conocidas aplicaciones de la informática en el ámbito educativo la constituye la denominada enseñanza asistida por ordenador. Desde los año 60 una de las preocupaciones fundamentales de la informática educativa ha sido la construcción de programas para la enseñanza. Estos han adoptado modalidades muy diversas. Podemos encontrar programas centrados en la generación de ejercicios para la consecución de aprendizajes en que la práctica sistemática y continuada es fundamental, programas de tipo tutorial que asumen la transmisión de información en un área de conocimiento determinado, programas de simulación que muestran al sujeto aspectos que difícilmente podrían ser observados en la realidad permitiendo además la modificación de parámetros, etc.

La EAO comienza a tener una historia bastante larga y por consiguiente el volumen de software es también considerable. Ello dificulta la realización de valoraciones globales sobre su calidad tanto a nivel técnico como pedagógico. No obstante, hay que tener en cuenta que dentro de las aplicaciones de la informática en la enseñanza la EAO ha sido, hasta el momento, el prototipo de la enseñanza tradicional. Es un hecho conocido que los primeros programas realizados tuvieron sus raíces en la enseñanza programada y en el uso de las máquinas de enseñar. La teoría del condicionamiento operante junto con las aportaciones sobre la naturaleza de las respuestas del alumno en base a la concepción ramificada de Crowder ha sido el modelo de la organización, estructuración y transmisión del conocimiento en la $\mathrm{EAO}$. Es evidente que la tecnología empleada para la construcción de este tipo de software tiene muchas limitaciones. En primer lugar, hay que tener en cuenta que la capacidad de almacenamiento de los ordenadores utilizados en la enseñanza es bastante limitada. Como consecuencia, los programas elaborados no pueden ser excesivamente potentes. En segundo lugar, hay que tener presente que los lenguajes de programación utilizados están basados en el procesamiento numérico y secuencial y ello determina también la posibilidad de construir programas muy abiertos. Por último, hasta hace poco tiempo la mayor parte de los lenguajes de autor comercializados sólo permitían construir programas con una estructura secuencial muy simple lo que añadido a la carencia de uso de animación y gráficos daba como resultado productos muy pobres desde el punto de vista tanto técnico como pedagógico. Este último aspecto es quizá el que más está cambiando en la actualidad. De hecho, existe ya una gran variedad de lenguajes de autor que permiten la creación de un software mucho más interactivo, con uso de animación, gráficos, control de vídeodisco, etc. Incluso en algunos casos, como por ejemplo el programa Hypercard desarrollado para Macintosh, se han construido pequeños programas que procesan la información de forma similar a como lo hacen los sistemas expertos que describiremos más adelante. Este es el caso de Hyperx que permite construir sistemas expertos sencillos que pueden ser añadidos a cualquier otro software realizado con este mismo programa. En definitiva, la frontera entre los programas de EAO clásicos y los TI se va haciendo cada vez más borrosa debido a la mejora de las herramientas de producción se software. A 
pesar de ello, la mayor parte de los programas del mercado actual y muy especialmente los elaborados para la enseñanza básica presentan bastantes limitaciones por lo que muchos de ellos resultan de escasa calidad pedagógica.

A los programas de EAO se les critica fundamentalmente su incapacidad por mantener un diálogo abierto con el alumno. Siempre es el ordenador el que presenta la información y espera la respuesta del alumno sin poder éste adquirir otras información en cualquier momento de la interacción. Además del aspecto comunicativo, el software desarrollado sigue estructuras de transmisión de conocimiento de carácter secuencial previamente determinadas y, por consiguiente, los programas carecen de la capacidad de una adaptación real a las necesidades y al estilo de aprendizaje individual del alumno.

Hacia los años 70, algunos autores (Carbonell 1970, Sleeman 1973) comenzaron a pensar que esta forma de adaptación sólo podría lograrse incorporando al diseño de software técnicas procedentes del campo de la IA; así es como surgieron los denominados tutoriales inteligentes.

Tal y como hemos señalado, con los micromundos no se busca la creación de programas que actúen inteligentemente sino de medios de exploración que faciliten el desarrollo del alumno. Por el contrario, en el caso de los TI se pretende que sea el propio ordenador quien adopte una forma de conducta inteligente que permita controlar el proceso de aprendizaje del alumno. Así pues, «los TI son programas de ordenador que utilizan técnicas procedentes de la IA para representar el conocimiento y llevar a cabo una interacción con el alumno» (D. Sleeman - J. Brown, 1982, p. 1).

El objetivo fundamental de los TI es, pues, proporcionar una instrucción adaptada al alumno tanto por el contenido como en la forma, superando de esta manera algunos de los problemas más acuciantes del software educativo existente en la actualidad. Prodríamos plantear esta superación en términos comparativos señalando que mientras la EAO se ha presentado como un material didáctico más próximo al libro de tex to o al cuaderno de ejercicios, los sistemas inteligentes de EAO apuntan hacia una modelización del comportamiento próxima a la del profesor.

Cuando se hacen afirmaciones como las anteriores conviene matizar un poco pues puede transmitirse la sensación de que muy pronto toda la enseñanza estará contenida en programas que serán tan inteligentes como los profesores y que actuarán igual o mejor que ellos. De este modo, la tarea instructiva que éstos realizan desaparecería. En este sentido, es importante señalar que la realidad está todavía muy lejos de alcanzar tales propósitos. De este modo, mientras que existe un volumen muy considerable de programas de EAO no existen TI comercializados y utilizados en el marco escolar. Hasta el momento los productos di- señados se han mantenido como prototipos empleados exclusivamente a nivel exploratorio. Este es un aspecto que conviene tener presente porque en numerosas ocasiones al contraponer los objetivos y características de la EAO con los TI es posible generar unas expectativas excesivamente optimistas de la calidad de éstos últimos respecto a los primeros que no corresponderían exactamente a la realidad del material diseñado hasta el momento.

La EAO y los TI aunque aparentemente se sitúan en un tipo similar de aplicación de la informática en la enseñanza, en realidad constituyen dos tipos de aplicaciones entre las que se pueden apreciar bastantes diferencias. Nos centraremos a continuación en la descripción de algunas de ellas (O. Park et al, 1987, p. 31):

1. Origen. Tradicionalmente las investigaciones y el desarrollo de material para la EAO han surgido de los propios profesionales de la enseñanza. En contraste, los TI fueron diseñados inicialmente por investigadores procedentes del campo de las ciencias computacionales para explorar las capacidades técnicas de la IA en el proceso de enseñanza-aprendizaje. El foco de los proyectos de los TI ha sido sobre todo en los aspectos técnicos más que en el dominio de un área de conocimiento o en los meramente instructivos. No obstante, conviene señalar que esta área de trabajo es, en la actualidad, fundamentalemente interdisciplinar agrupando especialistas en ciencia congnitiva, IA, eduación, psicología y lingüística.

2. Bases teóricas. Como hemos mencionado anteriormente, los programas de EAO se sitúan en el contexto de la enseñanza programada y los planteamientos de la psicología desarrollada por Skinner. Por el contrario, los TI parten de las aportaciones de la psicología cognitiva. Por ello, mientras que en los programas de EAO se tiende a controlar mucho las respuestas de los alumnos y los mecanismos de feedback para que éstos lleguen a dar la respuesta esperada, en los TI se está mucho más interesado en ir evaluando las diferentes respuestas dadas por el alumno a lo largo de su interacción con el ordenador para detectar aspectos tales como el estilo del aprendizaje del alumno, su grado de interés, comprensión, etc. En definitiva, los proyectos en esta área han mostrado un mayor interés por detectar los procesos internos del alumno que surgen en una tarea específica de enseñanza-aprendizaje.

3. Estructuración y funciones. En la mayoría de los programas de $\mathrm{EAO}$ todos los componentes son almacenados e incorporados en una única estructura y aunque algunos sistemas tienen módulos sèparados, la actuación de los componentes está predeterminada algorítmicamente. Es decir, toda la información que se pretende transmitir está fragmentada en pequeñas unidades y su secuencia viene definida por las respuestas de los alumnos. De este modo, si un alumno desea obtener una deter- 
minada información es posible que no pueda hacerlo directamente hasta que no haya contestado todas las respuestas previstas por el programa. Los TI tienden a la organización de la información en base a sistemas de búsqueda heurística que permitan llegar a una mezcla de iniciativas entre el ordenador y el alumno. Se intentan construir módulos separados a los que pueda accederse sin necesidad de ver todo el programa previamente. En resumen, con un programa clásico de $\mathrm{EAO}$, todos los alumnos acabarían viendo la misma información en un orden muy similar mientras que con un TI, éstos podrían acceder a partes del programa sin necesidad de trabajar todos los materiales que el tutorial tiene programado. La secuenciación estaría en función de la elección del alumno y del análisis del conjunto de respuestas dadas por éste.

4. Métodos de estructuración del conocimiento. $\mathrm{El}$ conocimiento en los programas de EAO suele estar estructurado tal y como se ha señalado anteriormente, de forma algorítmica. La tarea principal está formada por un conjunto de subtareas que van sucediéndose secuencialmente. Los TI recogen las técnicas de representación del conocimiento utilizadas en IA. Ello complica mucho más la estructura de los contenidos pero proporciona un tipo de conocimiento que no necesariamente ha de estar organizado de forma secuencial sino que es posible el acceso a partes específicas del mismo a través de estrategias heurísticas.

5. Métodos de modelización del alumno. La EAO utiliza un modelo de alumno basado en el juicio binario sobre las respuestas dadas por éste a las preguntas presentadas por el programa. Las acciones posteriores se encuentran en función del acierto o error de dicha respuesta sin tener en cuenta la historia particular del alumno. Así pues, la diferencia fundamental entre los modelos usados por los programas de EAO y los TI es que mientras los primeros evalúan la última respuesta, los segundos intentan evaluar todas las respuestas del alumno durante la ejecución del programa.

6. Formato de la instrucción. En el caso de los programas de EAO el formato de la instrucción se presenta básicamente a través de cuatro modalidades distintas: a) Tutorial. El ordenador actúa como tutor èn una determinada temática. b) Práctica y ejercitación. El ordenador proporciona un conjunto de ejercicios para permitir que el alum. no adquiera una determinada habilidad. c) Simulación. El ordenador se convierte en un laboratorio con el cual el usuario puede experimentar situaciones que resultan difíciles de manejar en la realidad. d) Juegos. Esta última categoría agrupa a un conjunto de programas que adoptan la forma de juego como medio de enseñanza.

Hasta el momento los TI se han centrado en la confección de programas próximos a los modelos tutoriales y de práctica y ejercitación. Más adelante retomaremos este aspecto para ver con más detalle algunos ejemplos de TI.
7. Hardware $y$ software. Los programas de $\mathrm{EAO}$ funcionan en cualquier tipo de ordenador personal e incluso en ordenadores con pocas posibilidades de almacenamiento de información. La mayor parte de estos programas han sido construidos mediante lenguajes de propósito general (BA. SIC, PASCAL, etc.) o bien a través de lenguajes de autor. En contraste, la mayoría de los TI precisan de un hardware mucho más potente que posea más capacidad de almacenamiento de la que tiene un ordenador doméstico. Para la construcción del software es preciso el uso de lenguajes específicos de IA tales como el LISP o el PROLOG o bien la utilización de herramientas de ayuda para la construcción de sistemas expertos especialmente diseñadas para tal fin (shells).

\section{Características generales de los TI}

Los TI poseen cuatro componentes fundamentales (J. Hartley - D. Sleeman, 1973): el módulo del experto que ha de poseer el conocimiento que el sistema trata de impartir al alumno, el módulo del alumno que indica cómo se está produciendo el aprendizaje del alumno, el módulo tutorial que especifica cuándo el sistema debe presentar el material al alumno y el módulo de comunicación que permite la interacción entre el sistema y el alumno. La IA aplicada a la educación se ha enfrentado desde sus inicios con la resolución de estos cuatro componentes y la clave del éxito de la construcción de este tipo de programas radica precisamente en un mayor dominio de la construcción de estos módulos. Dominio que hace referencia a los aspectos puramente técnicos pero también a los aspectos pedagógicos que condicionan el contenido de dichos módulos.

La aplicación primaria de la IA en la construcción de programas de enseñanza se centra pues en el dominio del conocimiento y en la representación de métodos de enseñanza. Idealmente, esta empresa supone tener una teoría de la enseñanza y de la naturaleza del conocimiento que debe ser enseñado. Un aspecto fundamental en la construcción de TI es que el ordenador debe contener todo el conocimiento que se posee sobre el tema a enseñar y sobre cómo debe enseñarse en cada momento según la actuación del alumno. Generalmente, este tipo de conocimiento se basa en el diagnóstico a través de reglas y relaciones causales y es usado durante los programas tutoriales para generar soluciones cuando se compara el estado real e ideal del comportamiento del alumno. $\mathrm{La}$ combinación de una base de conocimientos que contengan el conocimiento a transmitir y un intérprete que decida cuándo aplicarlos constituye un sistema experto.

Un sistema experto es «un sistema informático que incorpora, en forma operativa, el conocimiento de una persona experimentada, de forma que es capaz tanto de responder como esta persona co- 
mo de explicar y justificar sus respuestas» (J. Ganascia, 1985, p. 1213). Así, un sistema experto es un programa que utiliza una base de conocimientos acerca del dominio de una tarea y un motor de inferencias que permite establecer razonamientos para alcanzar la solución del problema. De este modo, todo TI ha de incorporar un sistema experto que contenga los conocimientos necesarios sobre cómo organizar la información y cuándo transmitirla al alumno.

\section{Tipos de TI}

Dada la complejidad que supone la construcción de un TI, tanto desde el punto de vista técnico como del propio diseño pedagógico, los programas construidos hasta el momento han adoptado forma diferentes resaltando modos específicos de aplicación. La mayor parte de autores coinciden en distinguir tres tipos (P. Duchastel, 1988. P. Ercoli - R. Lewis, 1988): a) aquellos que pretenden proporcionar instrucción al alumno a través del diagnóstico y detección de errores, b) los que se centran en el entrenamiento de una tarea y, c) los que desarrollan métodos de comunicación bidireccional para lograr guiar el proceso de aprendizaje del alumno a través del diálogo con el sistema. No obstante, las frontera entre estos tres tipos no siempre son claras. En este sentido, opinamos que siguiendo la terminología utilizada al hablar de los tipos de programas de la EAO es más claro afirmar que la mayor parte de TI son programas tutoriales y/o programas de práctica y ejercitación. La diferencia entre los programas estriba en que mientras unos destacan el diagnóstico y la detección de errores otros destacan la asistencia o ayuda para adquirir un determinado tipo de aprendizaje. Pondremos a continuación dos ejemplos que muestran esta doble diferenciación.

a. Un TI para ayudar a los niños a adquirir destrezas en el cálculo aritmético.

Desde principios de los ochenta (T. O'Shea et $a l, 1988)$ han estado diseñando una serie de herramientas que tienen por objeto ayudar al niño a adquirir destrezas básicas para la realización de las cuatro operaciones aritmáticas elementales. El programa intenta dar una ayuda individualizada al alumno de forma tal que lo primero que éste hace es detectar el grado de conocimiento del alumno a través de los diferentes tipos de errores que éste realiza. Es importante destacar que a diferencia de los programas de EAO, este tutorial diferencia entre muchos tipos de errores distintos y para cada uno de ellos ofrece un tipo de remedio distinto. Los resultados logrados son los siguientes:

1. Un conjunto de diagnósticos sobre el nivel del alumno.

2. Una extensa taxonomía de errores de las cuatro funciones aritméticas básicas.

3. Un conjunto de reglas de producción para las cuatro operaciones aritmáticas.
Este tutorial es pues un programa de práctica y ejercitación que se fundamenta en el diagnóstico y la detección de errores.

b. Un TI para ayudar a adquirir destrezas sobre la composición de textos escritos.

Algunos procesadores de texto incorporan programas de ayuda que permiten realizar correcciones ortográficas. El programa que están desarrollando M. Sharples y C. O'Malley (1988) pretende ampliar esta función y conseguir incorporar un tutorial que ayude a componer textos escritos que presenten un cierto grado de complejidad. Para utilizar este programa es preciso haber adquirido unas estrategias elementales de escritura. El tutorial pretende ayudar en la manipulación del material desde tres puntos de vista distintos:

1. La estructura. Hace referencia a las relaciones entre las diversas ideas o fragmentos del texto.

2. La linealidad. Se refiere a cómo transformar la estructura real del texto (las interrelaciones conceptuales) en un texto lineal.

3. La organización. Aquí el término organización hace referencia a la forma y no al contenido del texto. Es decir, cómo presentar la información, cómo introducir gráficos, esquemas, etc.

En este caso, el programa enfatiza la asistencia o ayuda para adquirir un determinado tipo de aprendizaje sobre el diagnóstico y detección de errores.

\section{Herramientas de ayuda dirigidas al profesor}

Se ha mencionado con anterioridad cómo los sistemas expertos pueden ser una buena herramienta de ayuda en el análisis y resolución de problemas complejos. Esta ayuda puede encaminarse hacia la construcción de tutores inteligentes, tal y como hemos visto, pero también puede pensarse en la producción de programas que asesoren y ayuden al profesor en tareas de diseño, planificación y organización de sus propias actividades. De hecho, esta idea ya hace un tiempo que se encuentra presente y existen varios equipos de trabajo investigando sobre el tema. A modo de ejemplo, $\mathrm{S}$. Wood de la Universidad de Sussex (S. Wood, 1988.a, 1988.b) dirige un proyecto cuyo objetivo es construir un sistema experto que permita guiar y aconsejar al profesor en su tarea instructiva. Este sistema experto pretende incorporar una serie de procesos que habitualmente aparecen en una situación de enseñanza-aprendizaje en el aula tales como: el control, la motivación, la comunicación, las relaciones entre profesor y alumno, la memorización, etc. Y a partir de ellos, según la situación descrita por el profesor, aconsejarle en la práctica de una determinada lección.

Otro proyecto que puede servir de ejemplo es el que se está desarrollando en la Universidad de Utah (EEUU) dirigido por D. Merrill (1989). En 
este caso, se pretende diseñar un conjunto de sistemas expertos que permitan asesorar en el diseño instructivo. Este sistema es pues algo más específico que el anterio ya que no se ocupa de la totalidad de la acción del profesor sino únicamente de cómo éste debe organizar el conocimiento para enseñar una determinada temática. De hecho, los primeros prototipos desarrollados por este equipo se han centrado en la creación de un programa que dé unas ideas generales sobre el diseño de la lección a transmitir según la teoría instructiva desarrollada por el propio Merrill y que viene a ser una adaptación de la teoría de la instrucción de Gagné.

Los programas dirigidos al asesoramiento del profesorado son, en nuestra opinión, un tipo de material de gran interés pero al igual que los TI, su construcción es una tarea muy compleja y los resultados que se han obtenido hasta el momento presentan bastantes limitaciones. Una de las causas que han hecho difícil el desarrollo de TI es precisamente el hecho de tener que utilizar técnicas y lenguajes propios de la IA. Una de las consecuencias de este hecho, es que los profesionales de la educación no han tenido demasiadas oportunidades para participar en el desarrollo de este tipo de materiales. Afortunadamente este aspecto comienza a cambiar sobre todo gracias a la construcción de herramientas de ayuda para la programación de sistemas expertos. Estas herramientas denominadas shells permiten construir sistemas expertos sin necesidad de conocer un lenguaje de programación específico. De hecho, este material es comparable a los lenguajes de autor en relación a la EAO.

Una shell proporciona una estructura vacía que es preciso llenar con el contenido que se pretende transmitir y con los razonamientos que el sistema debe manejar para decidir qué conocimiento debe transmitir y cuándo debe hacerlo.

Cada vez se han ido construyendo shells más sofisticadas y hay que tener en cuenta que aunque no es preciso conocer un lenguaje de programación su utilización tampoco es una tarea sencilla. La ventaja, en nuestra opinión, es que puede ha. cer más comprensible el proceso de construcción del programa y, por consiguiente, es más fácil crear grupos de producción de TI en los que participen profesionales tales como psicólogos, pedagogos y profesores. No obstante, también hay que tener en cuenta que además de las limitaciones a nivel de programación existen también problemas a la hora de poder incorporar en la máquina los razonamientos e inferencias adecuadas para llevar a cabo una determinada enseñanza o ayuda. De hecho, la diferencia fundamental entre la aplicación de la IA en el campo de la medicina o las finanzas y la educación estriba en la dificultad de concretizar y detallar los conocimientos psicopedagógi$\cos$ que el experto aplica en una determinada realidad.

\section{EL FUTURO DE LA IA APLICADA A LA EDUCACION}

En definitiva, pensamos que el futuro de la IA aplicada a la educación pasa por el desarrollo de dos aspectos que aunque, a nivel teórico, están diferenciados, a un nivel práctico esta diferenciación no puede ser excesiva. Nos estamos refiriendo a los desarrollos de tipo técnico y los desarrollos de tipo pedagógico.

En cuanto al desarrollo de tipo técnico, consideramos que el futuro de la construcción de un software de mayor calidad pasa por una mayor producción de shells (B. Gros, 1989) que faciliten la construcción de estos programas educativos. En la actualidad es ya posible comenzar a construir productos mediante el uso de shells de tipo general pero, consideramos muy interesante, la creación de herramientas pensadas específicamente para el sector educativo.

En cuanto al desarrollo pedagógico, pensamos que se precisa una mayor investigación básica en el terreno de los procesos de enseñanza-aprendizaje en dominios específicos de conocimiento. Pero además de este tipo de investigación, o mejor junto a ella, es precisa una mayor formación de los profesionales de la educación para que éstos puedan participar en el desarrollo de este tipo de programas. Sin una incorporación de los conocimientos pedagógicos no es posible la evolución en la construcción de TI, pero tampoco es posible dicha evolución si los profesionales que han de participar en ella no poseen unos conocimientos técnicos adecuados.

FIGURA 1

HERRAMIENTAS DIRIGIDAS

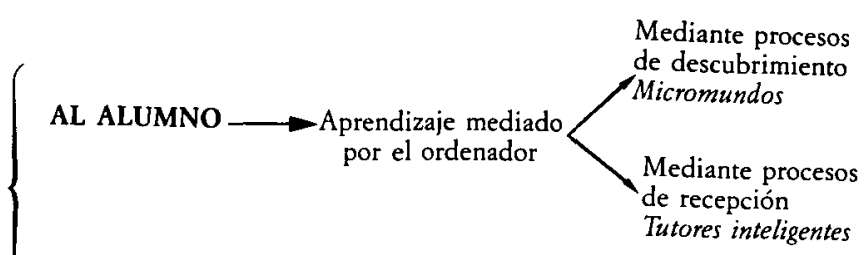

AL PROFESOR $\longrightarrow$ Ayuda a la planificación, diseño y organización de la tarea docente 


\section{Referencias}

Duchaster, P. (1988). «Models for AI in educational tutorin systems», en P. Ercoli - R. Lewis: Artificial inteligence in education, Amsterdam, North-Holland, 1988, pp. 17-29.

Gros, B. (1987). Aprender mediante el ordenador, Barcelona, PPU, 1987.

Gros, B. (1989). Análisis de berramientas de ayuda para la construcción de sistemas expertos aplicados al ámbito educativo, Barcelona, ICE de la Universidad de Barcelona, Informe interno, 1989.

Harmon, P. et al. (1988). Expert Systems. Tools \& Applications, New York, John Wiley \& sons, 1988 G. KeARSKEY (Ed.) (1987), Artificial Intelligence E Instruction, Reading, Addison-Wesley, 1987.

LAWLER, R. (1985). Computer experience and congnitive development, New York, Ellis Horwood, 1985.

Lawler, R. - YAzDANI, M. (Ed.) (1988). Artificial Intelligence and Education, Norwood, Ablex Publishing, 1988.

Merrill, D. - Li, Z. (1989). «An Instructional Design Expert System», Joumal of Domputer Based Instruction, Vol. 16, n. 3 , pp. 95-101.

O'sHea, T. et al. (1988). «Design choices for an intelligent arithmetic tutor» en J. SELF (Ed.), Artificial intelligence and buman learning, London, Chapman \& Hall, 1988, pp. 257-275.

Papert, S. (1973). «Uses of technologie to enhance education», Logo Memo, n. 8.

Papert, S. (1980). Minsdstorm. Children. Computers and Powerful Ideas, New York, Basic Books, 1980.

PAPERT, S. (1988). «Microworlds: transforming education», en R. LAWLER - M. YAzDANI (Ed.): Artificial Intelligence and Education, Norwood, Ablex Publishing, pp. 79-94.

$P_{A R K}, O$. et al. (1987). «Intelligent CAI: Old wine in new bottles, or a new vintage?", en G. KEARSKEY (Ed.): Artificial Intelligente \& Instruction, Reading, Addison-Wesley, pp. 11-56.

Rodríguez, L. - Rosello (1986). LOGO: de la tortuga a la inteligencia artificial, Madrid, Vector.

Sharples, M. - O'malley (1988). «A framework for the design of a writer's assistant" J. Self (Ed.), Artificial intelligence and buman learning, London, Chapman \& Hall, 1988, pp. 276-290.

Wood, S. (1988.a). «Expert Systems and Understanding Techar Education and Practice», en J. Calderhead (Ed.): Teacher's professional learning, London, Falmer Press, pp. 115-127.

Wood, S. (1988.b). «Trainee Teacher Support System: an expert system pro advesing trainee teachers on the classroom practice», Expert Systems, Vol. 5, n. ${ }^{\circ} 4$.

\section{La inteligencia artificial y su aplicación en la enseñanza. \\ Begoña Gross \\ CL\&E, 1992, 13, pp. $73-80$}

Resumen: El objetivo fundamental de este artículo es mostrar la influencia del desarrollo de la inteligencia artificial (IA) en el desarrollo de los programas informáticos aplicados en el ámbito educativo. Para ello, en primer lugar se describirán las metas y objetivos generales de las investigaciones en inteligencia artificial realizadas hasta la fecha a través de una comparación entre los sistemas de enseñanza asistida por ordenador (EAO) y los tutores inteligentes (TI).

Posteriormente se realiza un breve repaso a las diferentes aplicaciones de la IA en la educación centrándonos a continuación en el análisis de los aspectos más problemáticos que surgen en la construcción de los tutores inteligentes (TI) y los sistemas expertos. De este modo, se revisará el problema de la modelización del alumno y la representación del conocimiento así como de las herramientas a utilizar para la confección de sistemas expertos para la enseñanza

Datos sobre la autora: Begoña Gross es profesora titular en el Departamento de Teoría e Historia de la Educación de la Facultad de Pedagogía de la Universidad de Barcelona.

Dirección: Universidad de Barcelona. Facultad de Pedagogía. Departamento de Teoría e Historia de la Educación. C/. Baldiri i Reixac, s/n. 08028 Barcelona.

(C) De todos los artículos. Deberá solicitarse por escrito autorización de CL\&E y de los autores para el uso en forma de facsímil, fotocopia o cualquier otro medio de reproducción impresa. CL\&E se reserva el derecho de interponer las acciones legales necesarias en aquellos casos en que se contravenga la ley de derechos de autor. 